\title{
Percepção de regras e de confiança em redúzir o tempo de tela em adolescentes
}

\section{Rules perception and confidence in the ability to reduce screen time in adolescents}

\author{
Roseanne Gomes Autran ${ }^{1,2}$ \\ Cassiano Ricardo Rech ${ }^{3}$ \\ Jorge Mota ${ }^{1,2}$ \\ Maria Paula Santos ${ }^{1,2}$
}

\section{RESUMO}

O objetivo deste estudo foi analisar a associação entre o tempo de tela com a percepção de regras e a confiança em reduzir o tempo de tela em adolescentes. Participaram 358 adolescentes (58,2\% meninas), entre 14 e 18 anos, da cidade do Porto - Portugal. A percepção de regras e a confiança em reduzir o tempo de tela foram avaliadas por medidas autorreportadas através de instrumentos validados para esta população. Os adolescentes que percebem alguma regra por parte dos pais apresentam menor prevalência de exposição ao tempo de tela ( $\geq 2$ horas/dia) para uso da internet ( $R P=0,62$; IC95\%: 0,28-0,99). A moderada ( $R P=0,60$; IC95\%: 0,34-0,99) ou elevada $(\mathrm{RP}=0,51$; IC95\%: 0,27-0,96) confiança na capacidade de reduzir o tempo de tela foi associado de forma inversa com a prevalência de não cumprir as recomendações do tempo de tela para assistir TV e usar vídeo game/ computador. A percepção de regras e moderada ou elevada confiança na capacidade de reduzir o tempo de tela foram associados com menor proporção de adolescentes expostos a atividades sedentárias, tais como assistir TV e usar internet por $\geq 2$ horas/dia.

\section{PALAVRAS-CHAVE}

Estilo de vida sedentário; Adolescentes; Percepção; Associação.

\begin{abstract}
The aim of this study was examine the association between screen time with rules perception and confidence to reduce screen time in adolescents. Data were obtained from 358 adolescents $(58.2 \%$ girls) aged between 14 and 18 years old in the city of Porto - Portugal. To evaluate the amount time in screen time activities, as well as the confidence and rules perception we used validated self-reported surveys. Adolescents who perceived some rule have less likelihood to screen time ( $\geq 2$ hours/day) for Internet use $(P R=0.62 ; 95 \%$ IC: 0.28 to 0.99). The moderate $(R P=0.60 ; 95 \%$ IC 0.34 to 0.99) or bigh $(R P=0.51 ; 95 \%$ IC: 0.27 to 0.96$)$ confidence in the ability to reduce screen time was associated inversely with prevalence to meet the recommendations of screen time to watch $T V$ and play video game/computer. There was an association in the capacity to reduce screen time between adolescents who perceive some rules and showed moderate to bigh confidence and the exposed time sedentary activities, such as, watching TV and Internet utilization for more $\geq 2$ bours/day.
\end{abstract}

\section{KEYWORDS}

Sedentary life-style; Adolescents; Perception; Association.
Rev Bras Ativ Fis Saúde p. 690-699 $\mathrm{DOI}$

http://dx.doi.org/10.12820/rbafs.v.19n6p690

1 Faculdade de Desporto da Universidade do Porto. Porto, Portugal.

2 Centro de Investigação em Atividade Física, Saúde e Lazer (CIAFEL). Porto, Portugal.

3 Universidade Federal de Santa Catarina. Florianópolis, Brasil 


\section{INTRODUÇÃO}

Recomendações internacionais enfatizam que crianças e adolescentes não deveriam destinar mais do que duas horas por dia em atividades sedentárias relacionadas ao tempo de tela, como assistir televisão (TV), jogar vídeo game, usar o computador ou internet ${ }^{1}$. Existe uma relação direta entre o tempo gasto em atividades relacionadas com o tempo de tela e uma maior chance em desenvolver obesidade e doenças cardiovasculares na adolescência ${ }^{2}$.

Além disso, quanto mais elevado o tempo de tela na adolescência, maior o risco de obesidade na vida adulta ${ }^{3}$ independente do nível de atividade física ${ }^{4}$ Apesar destas evidências, aproximadamente 70\% dos adolescentes Europeus relatam passar mais de três horas por dia assistindo TV ${ }^{5}$, sendo que em meninas Portuguesas este valor é superior a $75 \%{ }^{6}$. Estudo realizado em jovens Portugueses, entre dois e 13 anos, observou uma relação direta entre o tempo gasto em assistir TV e marcadores de adiposidade, indicando que quanto maior o tempo de permanência neste comportamento maior os marcadores de adiposidade ${ }^{7}$. Nos Estados Unidos, um terço dos adolescentes ultrapassa a recomendação do tempo de tela ${ }^{8}$.

Investigações sobre o comportamento sedentário em adolescentes têm focado especialmente atividades relacionadas ao tempo de tela, por exemplo, assistir TV. Entretanto, menor atenção tem sido atribuída a outros potenciais comportamentos sedentários, como o tempo gasto no uso de vídeo game, computador e internet ${ }^{9}$. Além disso, poucos estudos analisaram os aspectos associados a estes comportamentos de forma independente para cada tipo de uso. Compreender os fatores correlatos a estes comportamentos pode auxiliar no desenvolvimento de estratégias para a redução do tempo de permanência em comportamento sedentário ${ }^{10}$, pois as intervenções propostas até o momento se mostraram pouco efetivas.

A percepção de regras para o uso de equipamentos eletrônicos e a confiança na capacidade em reduzir o tempo de tela, são aspectos emergentes e apontados como meios possivelmente eficazes para reduzir a exposição a esse comportamento em jovens ${ }^{11,12}$. Em crianças, a percepção de regras para uso de equipamentos eletrônicos, como por exemplo, limitar o tempo de uso e retirar a TV do quarto, mostrou-se eficiente para reduzir o tempo de tela ${ }^{11}$. Além disso, evidências indicam que a percepção de confiança na capacidade de reduzir a exposição ao tempo de tela é inversamente associada a esse comportamento ${ }^{12}$. A confiança é considerada um elemento chave da teoria sociocognitiva e enfatiza que a crença na capacidade de controle do comportamento é essencial para a adoção e manutenção do mesmo ${ }^{13}$. Porém, poucos estudos investigaram a relação entre a confiança na capacidade de reduzir o tempo de tela em adolescentes, considerando os diferentes tipos de comportamentos relacionados ao tempo de tela.

O objetivo deste estudo foi analisar a associação entre o tempo de tela com a percepção de regras e a confiança em reduzir o tempo de tela em adolescentes.

\section{MÉTODO}

\section{População e Amostra}

Os dados deste estudo fazem parte do projeto SALTA - "Suporte do Ambiente 
para o Lazer e Transporte Ativo" - que avaliou a influência das características do ambiente físico e social na atividade física em adolescentes na cidade do Porto - Portugal ${ }^{14}$.

Inicialmente todas as escolas $(\mathrm{n}=11)$ do Ensino Secundário do Município do Porto, Portugal, foram convidadas, por email, a participar do estudo. Os Diretores das Escolas receberam informação sobre o estudo. Destas, apenas três escolas aceitaram participar do estudo. A baixa taxa de adesão foi devido as mesmas encontrarem-se em um período de melhoria das condições estruturais das escolas públicas Portuguesas e também pela decorrência da reestruturação na organização do Sistema de Ensino Público Português.

Nas três escolas que participaram do estudo, 38 turmas (1,098 alunos) referentes ao $9^{\circ}, 10^{\circ}, 11^{\circ}$ e $12^{\circ}$ ano escolar no ano letivo de 2012/2013 foram selecionadas. Foi enviada uma carta para os pais dos adolescentes explicando os objetivos do estudo. Dos alunos contactados, 518 não retornaram as autorizações e 194 recusaram-se a participar das avaliações. Deste modo, 386 alunos entregaram a carta de consentimento assinada pelos pais concordando com a participação nas avaliações. Destes, 28 adolescentes foram excluídos por apresentarem questionários incompletos. Os adolescentes consentiram verbalmente a sua participação, antes da realização das avaliações. Os procedimentos utilizados seguiram os princípios da Declaração de Helsinki na investigação com Seres Humanos e foram aprovados pelo Comité de Desenvolvimento Curricular e da Divisão de Inovação (Ministério da Educação e Ciência do Governo Português).

O poder estatístico do tamanho da amostra foi calculado através do programa $\mathrm{G}^{*}$ Power, versão 3.0.5 ${ }^{(15)}$ que mostrou um poder estatístico superior a $80 \%$ para uma análise a posteriori, com um alpha=0,05 e uma amostra de 358 escolares e uma associação (RP) esperada de 1,2.

\section{VARIÁVEIS DO ESTUDO}

\section{Tempo de Tela}

O tempo de tela foi obtido através de uma entrevista individual semiestruturada com o adolescente. $\mathrm{O}$ instrumento incluiu o tempo em que o adolescente assistia TV, jogava vídeo game/computador e usava internet em um dia da semana. Utilizaram-se as seguintes questões: 1) Quanto tempo, por dia, você assiste TV? 2) Quanto tempo, por dia, você passa jogando/usando vídeo game/computador? 3) Quanto tempo, por dia, você usa internet? As opções de respostas foram: não uso, 1-15 minutos, 16-30 minutos, 31-60 minutos, 2 horas, 3 horas ou 4 horas ou mais. Para todas as questões consideraram-se apenas os dias de semana e não finais de semana. Os adolescentes que referiram passar duas ou mais horas/dia em tempo de tela foram classificados como " $n \tilde{a} 0$ cumpriam as recomendação" e aqueles que passaram menos de duas horas/dia, foram classificados como "cumpriam as recomendações" para o tempo de tela ${ }^{1}$.

A análise foi realizada separadamente para cada tipo de utilização (ex.: TV, vídeo game/computador e internet). Os entrevistadores foram treinados para identificar possíveis respostas sobrepostas (ex.: quando o adolescente reportava o mesmo tempo em diferentes tipos de uso). As questões utilizadas para medida de tempo de tela são fundamentadas em estudos anteriores sobre o tema ${ }^{17,18}$. 


\section{Confiança na capacidade de reduzir o tempo de tela}

A confiança na capacidade de reduzir o tempo de tela, foi avaliada através de uma escala composta por sete itens com cinco pontos de opções de resposta ("estou certo que não posso", "acho que não posso", "não sei se posso", "acho que posso", "estou certo que posso").

Os adolescentes foram questionados sobre qual a confiança na capacidade de lidar com questões relacionadas com o controle do tempo de tela nas seguintes situações: 1) "estou certo que consigo desligar a televisão mesmo quando está a passar um programa do meu agrado"; 2) "estou certo que consigo limitar o tempo que estou online no computador (ex.: navegar na internet, enviar emails) a uma horal dia"; 3) "estou certo que consigo sair do local em que a televisão está ligada, mesmo que outros fiquem lá assistindo"; 4) "estou certo que consigo planejar com antecedência o tempo que vou assistir os programas durante a semana"; 5) "estou certo que consigo, em vez de me sentar para ouvir música, ouvir enquanto estou realizando atividade física (ex.: a caminhar, a dançar)"; 6) "estou certo que consigo estabelecer limites relativamente a quanto tempo penso estar no telefone ou trocando mensagens com os meus amigos"; 7) "estou certo que consigo limitar a duas horas/dia o tempo em que assisto televisão/ vídeo".

Os itens que compuseram a escala apresentaram cargas fatoriais $(\geq 0,5)$ e alfa de Cronbach $(\geq 0,67)$ significativos ${ }^{16}$. A análise fatorial confirmatória demonstrou índices de ajuste satisfatórios (Chi-square: 17.390; p=0.135; $\mathrm{CMIN} / \mathrm{DF}=1.449 ; \mathrm{NFI}=0.923 ; \mathrm{CFI}=0.974$ e $\mathrm{RMSEA}=0.042$ ) para a escala. Para a análise, o valor de cada item foi somado e o escore final categorizado de acordo com a distribuição em tercil.

\section{Percepção de regras para reduzir o tempo de tela}

Para analisar a percepção dos adolescentes em relação às regras dos pais para o uso de equipamentos eletrônicos, utilizou-se uma escala composta por três itens. Os adolescentes foram questionados sobre as seguintes regras: 1) não permitem assistir televisão/DVD/computador antes de realizar as tarefas escolares; 2) não permitem usar a internet sem autorização; 3) estipulam que eu não deva assistir mais do que duas hora/dia de televisão/DVD/computador.

Utilizou-se uma escala dicotómica ("não" versus "sim") para a ausência ou presença de regras. Os itens que compuseram a escala apresentaram cargas fatoriais $(\geq 0,7)$ e alpha de Cronbach $(\geq 0,57)$ significativos ${ }^{16}$. A análise fatorial confirmatória demonstrou índices de ajuste satisfatórios (Chi-square:3.352; $\mathrm{p}=0.067 ; \mathrm{CMIN} / \mathrm{DF}=3.358 ; \mathrm{NFI}=0.961 ; \mathrm{CFI}=0.972 ; \mathrm{RMSEA}=0.051)$ para a escala.

\section{Aspectos sociodemográficos e de saúde}

Foram coletadas informações sociodemográficas de gênero (meninos/meninas), idade (anos). Além disso, a renda familiar foi obtida através do Sistema de Registo Administrativo das Escolas e classificado em nível A (mais elevado) e B (mais baixo). Também foi analisado nível educacional da mãe, sendo classificada de acordo com os critérios do Ministério Português de Educação: baixo (até 9 anos de estudos), médio (9-12 anos de estudos), alto (mais de 13 anos de estudos) ${ }^{19}$.

As medidas de peso e estatura foram avaliadas através de uma balança Tanita (Inner Scan BC 532, Tanita. Tokyo, Japan) e um estadiometro portátil 
(Crymych Pembrokeshire, UK). A partir das medidas de peso e estatura foi calculado o índice de massa corporal e o mesmo classificado em peso normal, sobrepeso e obeso, com valores específicos para gênero e idade ${ }^{20,21}$.

\section{Análise dos dados}

A análise da validade fatorial confirmatória das escalas de regras e confiança em reduzir o tempo de tela foi realizada através do Mplus 6.1, utilizando o estimador para correlações categóricas ${ }^{16}$. A descrição das variáveis, estratificada por gênero, foi realizada através da distribuição de frequência absoluta e relativa e do teste qui-quadrado para proporções. Após a análise bivariada, foi testada a associação entre o tempo de tela e as variáveis de regras e confiança em reduzir o tempo de tela, ajustado para as variáveis de confusão (gênero, idade, ano escolar, escolaridade da mãe e IMC), por meio da regressão de Poisson com variância robusta. Foram testados modelos para três diferentes tipos de uso de tela (TV, jogar vídeo game/computador, uso internet), de modo independente, sendo que os adolescentes que relataram ter duas horas/dia ou mais de tempo de tela foram considerados como aqueles que "não cumprem a recomendação". A percepção de regras para utilizar equipamentos eletrônicos foi dicotomizada ("percebem alguma regra" versus "não percebem nenhuma regra") e a confiança na capacidade de reduzir o tempo de tela categorizada de acordo com o tercil de confiança (baixa, média, elevada). As análises foram realizadas no programa estatístico Stata 12.0 e o nível de significância de $5 \%$.

\section{RESULTADOS}

Particiaram do estudo 358 alunos (58,2\% meninas), entre 14 e 18 anos de idade. Não foram encontradas diferenças estatisticamente significativas entre o gênero e a idade dos participantes e não participantes do estudo.

Maior parte da amostra foi composta por adolescentes entre 16-17 anos (54,2\%), pertencentes ao décimo/décimo primeiro ano de escolaridade $(59,4 \%)$ e $14,8 \%$ com sobrepeso. A proporção de meninos que passam mais de duas horas/dia jogando vídeo game/computador e usando a internet foi maior quando comparada com meninas $(\mathrm{p}<0,05)$. Não houve diferença significativa em relação à proporção de adolescentes que assistem mais de duas horas/dia de TV em relação ao gênero (ver, tabela 1).

$\mathrm{Na}$ tabela 2, observa-se na análise univariada que adolescentes que percebem alguma regra para utilizar os equipamentos eletrônicos apresentam menor prevalência de exposição excessiva ao tempo de tela ( $\geq 2$ horas/dia) para uso da internet $(\mathrm{RP}=0,62 ; 0,28-0,99)$. Também observou-se que moderada $(\mathrm{RP}=0,60 ; 0,34-0,99)$ ou elevada $(\mathrm{RP}=0,51 ; 0,27-0,96)$ confiança na capacidade de reduzir o tempo de tela foi associada de forma inversa com a prevalência para cumprir as recomendações do tempo de tela para assistir TV e jogar vídeo game/computador.

Após o ajuste para as variáveis de confusão (gênero, idade, ano escolar, escolaridade da mãe e status de peso), a percepção de regras manteve-se associada com menor prevalência de exposição excessiva ao tempo de tela na internet (Tabela 3). Os adolescentes que percebiam alguma regra para o uso de equipamentos eletrônicos apresentaram menor prevalência de não cumprirem as recomendações de tempo de tela. $\mathrm{O}$ mesmo foi observado em relação à con- 
TABELA 1 - Características descritivas da amostra (Porto, Portugal, 2012; n=358).

\begin{tabular}{|c|c|c|c|c|c|c|c|c|}
\hline \multirow{2}{*}{ Variáveis } & \multirow{2}{*}{ Categorias } & \multicolumn{2}{|c|}{ Meninos } & \multicolumn{2}{|c|}{ Meninas } & \multirow[t]{2}{*}{$p$} & \multicolumn{2}{|c|}{ Total } \\
\hline & & $n$ & $\%$ & $n$ & $\%$ & & $n$ & $\%$ \\
\hline Sexo & & 209 & 58,4 & 149 & 41,6 & & 358 & 100 \\
\hline \multirow[t]{6}{*}{ Idade (anos) } & & & & & & 0,80 & & \\
\hline & 14 & 11 & 7,4 & 22 & 10,5 & & 33 & 9,2 \\
\hline & 15 & 28 & 18,8 & 41 & 19,6 & & 69 & 19,3 \\
\hline & 16 & 35 & 23,5 & 51 & 24,4 & & 86 & 24,0 \\
\hline & 17 & 49 & 32,9 & 59 & 28,2 & & 108 & 30,2 \\
\hline & 18 & 26 & 17,4 & 36 & 16,5 & & 62 & 17,3 \\
\hline \multirow[t]{5}{*}{ Ano escolar } & & & & & & 0,99 & & \\
\hline & 90 & 23 & 15,4 & 32 & 15,3 & & 55 & 15,4 \\
\hline & $10^{\circ}$ & 43 & 28,9 & 61 & 29,2 & & 104 & 29,1 \\
\hline & $11^{\circ}$ & 44 & 29,5 & 61 & 29,2 & & 105 & 29,3 \\
\hline & $12^{\circ}$ & 39 & 26,2 & 55 & 26,3 & & 94 & 26,3 \\
\hline \multirow[t]{5}{*}{ IMC } & & & & & & 0,38 & & \\
\hline & Baixo peso & 4 & 3,2 & 10 & 5,8 & & 14 & 4,7 \\
\hline & Normal & 92 & 73,6 & 133 & 77,3 & & 225 & 75,8 \\
\hline & Sobrepeso & 23 & 18,4 & 21 & 12,2 & & 44 & 14,8 \\
\hline & Obeso & 6 & 4,8 & 8 & 4,7 & & 14 & 4,7 \\
\hline \multicolumn{4}{|c|}{ Confiança em reduzir o tempo de tela } & & & 0.005 & & \\
\hline & Baixa & 42 & 40,4 & 40 & 30,1 & & 82 & 34,6 \\
\hline & Moderada & 41 & 39,4 & 40 & 30,1 & & 81 & 34,2 \\
\hline & Elevada & 21 & 20,2 & 53 & 39,8 & & 74 & 31,2 \\
\hline \multicolumn{4}{|c|}{ Percebe regras sobre o tempo de tela } & & & 0,70 & & \\
\hline & Não percebe & 69 & 64,5 & 85 & 62,5 & & 154 & 63,4 \\
\hline & Percebe & 38 & 35,5 & 51 & 37,5 & & 89 & 36,6 \\
\hline \multicolumn{4}{|c|}{ Tempo a assistir TV } & & & 0,27 & & \\
\hline & $<2$ horas/dia & 77 & 72,0 & 118 & 65,8 & & 195 & 69,4 \\
\hline & $\geqslant 2$ horas/dia & 40 & 28,0 & 46 & 34,2 & & 86 & 30,6 \\
\hline \multicolumn{4}{|c|}{ Tempo vídeo game/computador } & & & 0,001 & & \\
\hline & $0-2$ horas/dia & 76 & 65,0 & 151 & 92,1 & & 227 & 80,8 \\
\hline & $\geqslant 2$ horas/dia & 41 & 35,0 & 13 & 7,9 & & 54 & 19,2 \\
\hline \multirow[t]{3}{*}{ Uso internet } & & & & & & 0,004 & & \\
\hline & $0-2$ horas/dia & 69 & 59,0 & 115 & 70,6 & & 184 & 65,7 \\
\hline & $\geqslant 2$ horas/dia & 48 & 41,0 & 48 & 29,4 & & 96 & 34,3 \\
\hline
\end{tabular}

Teste Qui-Quadrado considerando $\mathrm{p}<0,005$

IMC: Índice de massa corporal $\left(\mathrm{kgm} /{ }^{2}\right)$

fiança na capacidade de reduzir o tempo de tela. Moderada e elevada confiança reduziram a prevalência de exposição excessiva ao tempo de tela em assistir TV.

\section{DISCUSSÃO}

Os resultados indicaram maior tempo de exposição aos jogos eletrônicos (vídeo game e computador) entre meninos ( $62,3 \%$ vs $42,1 \%$; $\mathrm{p}<0,05)$, corroborando com evidências da literatura ${ }^{22,23}$, que também apontam que meninas preferem outras atividades sedentárias, como conversar com amigos e ouvir 
TABELA 2 -Análise da associação bruta entre diferentes comportamentos sedentários e a percepção de regras e confiança em reduzir o tempo de tela em adolescentes.

\begin{tabular}{|c|c|c|c|c|c|c|c|c|c|}
\hline \multirow{2}{*}{$\begin{array}{c}\text { Variáveis } \\
\%\end{array}$} & \multicolumn{3}{|c|}{$\begin{array}{c}\text { Tempo TV } \\
\text { ( } 22 \text { horas } / \text { dial }\end{array}$} & \multicolumn{3}{|c|}{$\begin{array}{c}\text { Tempo vídeo game/PC } \\
\text { ( } \geq 2 \text { horas/dial }\end{array}$} & \multicolumn{3}{|c|}{$\begin{array}{l}\text { Tempo internet } \\
\text { ( } \geq 2 \text { horas/dia) }\end{array}$} \\
\hline & $\mathrm{RP}$ & IC95\% & $\%$ & $\mathrm{RP}$ & IC95\% & $\%$ & $\mathrm{RP}$ & IC95\% & \\
\hline \multicolumn{10}{|c|}{$\begin{array}{l}\text { Confiança em reduzir } \\
\text { TT }\end{array}$} \\
\hline Baixa & 40,0 & 1,00 & & 26,7 & 1,00 & & 36,5 & 1,00 & \\
\hline Moderada & 24,0 & 0,61 & $0,35-0,98$ & 16,0 & 0,73 & $0,37-1,42$ & 36,0 & 0,96 & $0,57-1,61$ \\
\hline Elevada & 20,3 & 0,51 & $0,27-0,93$ & 11,6 & 0,43 & $0,19-0,98$ & 23,2 & 0,59 & $0,32-1,08$ \\
\hline \multicolumn{10}{|c|}{ Regras para controle TT } \\
\hline Não percebe & 29,6 & 1,00 & & 19,7 & 1,00 & & 37,6 & 1,00 & \\
\hline Percebe & 28,0 & 0,98 & $0,60-1,59$ & 15,9 & 0,82 & $0,43-1,55$ & 23,2 & 0,62 & $0,37-0,99$ \\
\hline
\end{tabular}

RP: Razão de Prevalência. IC95\%: intervalo de confiança 95\%. TT: Tempo de Tela.

TABELA 3 - Análise da associação ajustada entre diferentes comportamentos sedentários e a percepção de regras e confiança em reduzir o tempo de tela em adolescentes.

\begin{tabular}{|c|c|c|c|c|c|c|c|c|c|}
\hline \multirow{2}{*}{$\begin{array}{c}\text { Variáveis } \\
\%\end{array}$} & \multicolumn{3}{|c|}{$\begin{array}{c}\text { Tempo TV } \\
\text { ( } \geqslant 2 \text { horas/dial }\end{array}$} & \multicolumn{3}{|c|}{$\begin{array}{c}\text { Tempo vídeo game/PC } \\
\text { ( } 22 \text { horas/dia) }\end{array}$} & \multicolumn{3}{|c|}{$\begin{array}{l}\text { Tempo internet } \\
(\geqslant 2 \text { horas } / \text { dial }\end{array}$} \\
\hline & $\mathrm{OR}$ & IC95\% & $\%$ & OR & IC95\% & $\%$ & $\mathrm{OR}$ & IC95\% & \\
\hline \multicolumn{10}{|c|}{ Confiança em reduzir TT } \\
\hline Baixa & 40,0 & 1,00 & & 26,7 & 1,00 & & 36,5 & 1,00 & \\
\hline Moderada & 24,0 & 0,60 & $0,34-0,99$ & 16,0 & 0,69 & $0,35-1,36$ & 36,0 & 0,96 & $0,57-1,61$ \\
\hline Elevada & 20,3 & 0,51 & $0,27-0,96$ & 11,6 & 0,64 & $0,27-1,48$ & 23,2 & 0,63 & $0,34-1,18$ \\
\hline \multicolumn{10}{|c|}{ Regras para controle TT } \\
\hline Não percebe & 29,6 & 1,00 & & 19,7 & 1,00 & & 37,6 & 1,00 & \\
\hline Percebe & 28,0 & 0,98 & $0,60-1,60$ & 15,9 & 0,85 & $0,45-1,62$ & 23,2 & 0,62 & $0,28-0,99$ \\
\hline
\end{tabular}

RP: Razão de prevalência. IC95\%: intervalo de confiança 95\%. TT: Tempo de Tela. Análise ajustada para sexo, idade, ano escolar, escolaridade da mãe e IMC.

música. No geral, a prevalência de adolescentes que despendem mais de duas horas/dia em frente a TV, no computador ou na internet foi de 32,5\%, 20,1\% e 33,9\%, respectivamente. Em relação ao objetivo principal deste estudo, observou-se que adolescentes que perceberam alguma regra e possuem moderada ou elevada confiança na capacidade em reduzir o tempo assistindo TV e usando a internet possuem menor prevalência de exposição excessiva ( $\geq 2$ horas/dia) a estes comportamentos. A relação inversa entre a percepção de regras e o uso da internet é descrita em estudos prévios que suportam a importância desta variável na adoção deste comportamento ${ }^{24}$.

Porém, diferente de outros estudos, observou-se uma relação significativa entre percepção de regras e o uso de TV e vídeo game/computador ${ }^{24}$. Apesar de não haver diferenças significativas entre os gêneros relativamente ao comportamento de assistir TV, estudos revelam que o tempo despendido nesta atividade e a utilização do computador induzem efeitos adversos na saúde de crianças e adolescentes, como a obesidade e outras doenças crônicas ${ }^{25,26}$.

A adolescência representa um importante período de transição e consolidação dos comportamentos para a vida adulta. Em relação ao tempo de tela em adolescentes entre os 11 e 15 anos, investigações demonstraram um aumento médio de 60 minutos/dia no tempo de tela, com ênfase no tempo gasto no uso do computador ${ }^{27}$. Assim, neste período, o ambiente familiar pode con- 
tribuir como um elemento regulador/limitador do tempo destinado às atividades sedentárias. Intervenções com adolescentes para reduzir o tempo de tela que focaram o apoio dos pais se mostram eficazes e pode ter efeitos positivos 28. A função de controle e regulação dos comportamentos, por parte dos pais, no sentido de orientar para escolhas mais saudáveis, pode contribuir para uma maior adoção destes comportamentos ${ }^{29}$.

Além disso, a confiança na capacidade de decisão sobre reduzir o tempo de tela associou-se com o tempo excessivo de tela. Estes resultados reforçam a importância da confiança em adotar um comportamento, o que gera impactos nas atitudes e ações. A crença na capacidade de realizar uma tarefa ou alterar um comportamento é caracterizada como autoeficácia ${ }^{30}$. A confiança em adotar determinado comportamento pode gerar uma expectativa positiva em relação a intenção em realizar esse comportamento, fortalecendo assim, a relação positiva entre a característica psicossocial representada pela confiança em reduzir o tempo em atividades sedentárias ${ }^{31}$.

No presente estudo, a confiança não foi associada com menor tempo no uso da internet, o que pode estar relacionado com o fato da internet estar cada vez mais disponível na sociedade. Em vários locais pode-se ter acesso à internet através do telefone e as redes sociais tornam-na cada vez mais atrativas, pois permitem a comunicação e a interação entre os adolescentes de forma rápida. Portanto, será cada vez mais desafiador limitar o tempo de tela neste tipo de atividade, principalmente fora do ambiente doméstico. Contudo, é no ambiente doméstico que a família desenvolve um importante papel, tanto de influência dos comportamentos, quanto no controle de todas as atividades sedentárias, uma vez que a maior parte das atividades sedentárias desenvolve-se em ambiente familiar.

O tempo de tela foi autorreportado, o que pode limitar a precisão das medidas. $\mathrm{O}$ delineamento transversal não permite estabelecer relação temporal entre as variáveis analisadas. Assim, não podemos enunciar relação causal nos resultados encontrados. A amostra utilizada sugere cautela em extrapolar os dados para outras regiões do país, por não corresponder a uma amostra representativa. Sendo assim, novos estudos abordando variáveis de interesses dos adolescentes e considerando uma amostra representativa são necessárias para o fortalecimento dos resultados encontrados. Entretanto, uma vez que os resultados vão de encontro a estudos europeus, permite-nos considerar que o comportamento sedentário entre os adolescentes requer urgente atenção quanto às políticas de intervenção que visem a diminuição do tempo gasto em atividades sedentárias. Não foram analisados os efeitos moderadores de variáveis sociodemográficas, como gênero, idade, condição socioeconômica, que podem mostrar associações específicas em subgrupos populacionais. Estas análises não foram realizadas devido ao tamanho da amostra não permitir força para as estratificações necessárias.

Sendo assim, pode-se concluir que os adolescentes que percebem regras e com moderada e elevada confiança em reduzir o tempo de tela apresentam menor prevalência de se exporem, em tempo excessivo, em atividades sedentárias, como assistir TV e usar a internet. Maior atenção deve ser destinada ao ambiente familiar e a aspectos psicossociais em adolescentes para melhor entender os correlatos do comportamento sedentário. 


\section{Fonte de Financiamento}

O Projeto SALTA foi financiado pela Fundação para a Ciência e Tecnologia (FCT), concessão PTDC/DES/099018/2008 - FCT/FCOMP-01- 0124-FEDER-009573. O Centro de Investigação em Atividade Física, Saúde e Lazer (CIAFEL) é suportado por Pest-OE/SAU/UI0617/2011.

\section{REFERÊNCIAS}

1. Barlow SE, Expert C. Expert committee recommendations regarding the prevention, assessment, and treatment of child and adolescent overweight and obesity: summary report. Pediatrics. 2007; 120 (4):164-92.

2. Mitchell JA, Rodriguez D, Schmitz KH, Audrain-McGovern J. Greater screen time is associated with adolescent obesity: a longitudinal study of the BMI distribution from Ages 14 to 18 . Obesity. 2013; 21(3):572-5.

3. Biddle SJ, Pearson N, Ross GM, Braithwaite R. Tracking of sedentary behaviors of young people: a systematic review. Prev Med. 2010; 51(5):345-51.

4. Nelson MC, Gordon-Larsen P. Physical activity and sedentary behavior patterns are associated with selected adolescent health risk behaviors. Pediatrics. 2006; 117(4):1281-90.

5. Hallal PC, Andersen LB, Bull FC, Guthold R, Haskell W, Ekelund U, et al. Global physical activity levels: surveillance progress, pitfalls, and prospects. Lancet. 2012; 380(9838):247-57.

6. Mota J, Ribeiro JC, Santos MP. Obese girls differences in neighbourhood perceptions, screen time and socioeconomic status according to level of physical activity. Health Educ Res. 2009; 24(1):98-104.

7. 7.Stamatakis E, Coombs N, Jago R, Gama A, Mourao I, Nogueira H, et al. Associations between indicators of screen time and adiposity indices in Portuguese children. Prev Med. 2013; 56(5):299-303.

8. Matthews CE, Chen KY, Freedson PS, Buchowski MS, Beech BM, Pate RR, Troiano RP. Amount of time spent in sedentary behaviors in the United States, 2003-2004. Am J Epidemiol. 2008; 167(7):875-81.

9. Vandelanotte C, Sugiyama T, Gardiner P, Owen N. Associations of Leisure-Time Internet and Computer Use With Overweight and Obesity, Physical Activity and Sedentary Behaviors: Cross-Sectional Study. J Med Internet Res. 2009; 11(3): e28.

10. Babey SH, Hastert TA, Joelle Wolstein J. Adolescent Sedentary Behaviors: Correlates Differ for Television Viewing and Computer Use. J Adolescent Health. 2012; 52(2013):70-6.

11. Tandon PS, Zhou C, Sallis JF, Cain KL, Frank LD, Saelens BE. Home environment relationships with children's physical activity, sedentary time, and screen time by socioeconomic status. Int J Behav Nutr Phys Act. 2012; 9(88).

12. Norman GJ, Schmid BA, Sallis JF, Calfas KJ, Patrick K. Psychosocial and environmental correlates of adolescent sedentary behaviors. Pediatrics. 2005; 116(4):908-16.

13. Bandura A. Self-efficacy: The exercise of control. 1997.

14. Pizarro AN, Ribeiro JC, Marques EA, Mota J, Santos MP: Is walking to school associated with improved metabolic health? Int J Behav Nutr Phys Act 2013; 10(12).

15. Faul F, Erdfelder E, Lang AG, Buchner A. G*Power 3: a flexible statistical power analysis program for the social, behavioral, and biomedical sciences. Behav Res Methods. 2007; 39(2):175-91.

16. Byrne BM. Structural equations modeling with Mplus: basics concepts, applications, and programming, 2012.

17. Ramirez ER, Norman GJ, Rosenberg DE, Kerr J, Saelens BE, Durant N, et al. Adolescent screen time and rules to limit screen time in the home. J Adolescent Health. $2011 ; 48(4): 379-85$.

18. Carlson SA, Fulton JE, Lee SM, Foley JT, Heitzler C, Huhman M. Influence of limitsetting and participation in physical activity on youth screen time. Pediatrics. 2010; 126(1):89-96. 
19. Education PMO. Estatuto sócio-económico familiar.2009. Disponível em: http://www. portugal.gov.pt/pt.aspx.> [2013 mar 27].

20. Cole TJ, Bellizzi MC, Flegal KM, Dietz WH. Establishing a standard definition for child overweight and obesity worldwide: international survey. BMJ. 2000 6;320 (7244):1240-3.

21. Cole TJ, Flegal KM, Nicholls D, Jackson AA. Body mass index cut offs to define thinness in children and adolescents: international survey. BMJ. 2007; 28;335(7612), 194.

22. Granich J, Rosenberg M, Knuiman M, Timperio A. Understanding children's sedentary behaviour: a qualitative study of the family home environment. Health Educ Res. 2010; 25(2):199-210.

23. Rey-Lopez JP, Vicente-Rodriguez G, Ortega FB, Ruiz JR, Martinez-Gomez D, De Henauw S, et al. Sedentary patterns and media availability in European adolescents: The HELENA study. Prev Med. 2010; 51(1):50-5.

24. Ramirez ER, Norman GJ, Rosenberg DE, Kerr J, Saelens BE, Durant N, et al. Adolescent screen time and rules to limit screen time in the home. J Adolesc Health. $2011 ; 48(4): 379-85$.

25. Tremblay MS, LeBlanc AG, Kho ME, Saunders TJ, Larouche R, Colley RC, et al. Systematic review of sedentary behaviour and health indicators in school-aged children and youth. Int J Behav Nutr Phys Act. 2011;8(98).

26. Biddle SJ, O'Connell S, Braithwaite RE. Sedentary behavior interventions in young people: a meta-analysis. Br J Sports Med. 2011; 45(11):937-42.

27. Hoehner CM, Brennan Ramirez LK, Elliott MB, Handy SL, Brownson RC. Perceived and objective environmental measures and physical activity among urban adults. Am J Prev Med. 2005; 28(2):105-16.

28. Bjelland M, Bergh IH, Grydeland M, Klepp KI, Andersen LF., Anderssen SA, Ommundsen Y, Lien N. Changes in adolescents' intake of sugar-sweetened beverages and sedentary behavior: Results at 8 month mid-way assessment of the HEIA study a comprehensive, multi-component school-based randomized trial. Int J Behav Nutr Phy. 2011; 8(63).

29. Salmon J, Timperio A, Telford A, Carver A, Crawford D. Association of family environment with children's television viewing and with low level of physical activity. Obes Res. 2005; 13(11):1939-51.

30. Bandura A. Social foundations of thought and action: a social cognitive theory: Englewood Cliffs, NJ US: Prentice-Hall, Inc.; 1986.

31. Contento IR, Koch PA, Lee H, Sauberli W, Calabrese-Barton A. Enhancing personal agency and competence in eating and moving: formative evaluation of a middle school curriculum--Choice, Control, and Change. J Nutr Educ Behav. 2007; 39(5):179-86.

\section{ENDERECO PARA}

CORRESPONDÊNCIA

ROSEANNE GOMES AUTRAN

Rua Dr. Plácido Costa, 91 - Paranhos-

Porto-Portugal. CP:4200-450

Telefone: (351) 918448995

E-mail: roseautranđgmail.com

RECEBIDO $\quad 11 / 05 / 2014$

REVISADO 14/10/2014 $09 / 12 / 2014$ APROVADO 09/12/2014 